\title{
Transplanted embryonic retinal stem cells have the potential to repair the injured retina in mice
}

\author{
Xia Feng ${ }^{1}$, Peng Chen ${ }^{1}$, Xin Zhao ${ }^{1}$, Jing Wang ${ }^{1}$ and Hong Wang ${ }^{2^{*}}$ (D)
}

\begin{abstract}
Background: Stem cell transplantation has been reported as one of the promising strategies to treat retinal degenerative diseases. But, the application and the role of retina stem cells (RSCs) in the treatment of patients with retinal degenerative diseases have not been fully revealed. This study aimed to investigate the potential role of transplantation of the embryo-derived RSCs into the vitreous cavity in repairing the damaged retina in mice.

Methods: RSCs were isolated from Kunming mice E17 embryonic retina and ciliary body tissues, and labeled with 5-bromo-2'-deoxyuridin (BrdU). Retinal optic nerve crush injury was induced in left eyes in male Kunming mice by ring clamping the optic nerve. The 6th -generation of BrdU-labeled RSCs were transplanted into the damaged retina by the intravitreal injection, and saline injected eyes were used as the control. Hematoxylin and eosin histological staining, and BrdU, Nestin and Pax6 immunostaining were performed. Electroretinogram (ERG) was used for assessing the electrical activity of the retina.
\end{abstract}

Results: Embryo-derived RSCs were identified by the positive stains of Pax6 and Nestin. BrdU incorporation was detected in the majority of RSCs. The damaged retina showed cellular nuclear disintegration and fragmentation in the retinal tissue which progressed over the periods of clamping time, and decreased amplitudes of a and $b$ waves in ERG. In the damaged retina with RSCs transplantation, the positive staining for BrdU, Pax6 and Nestin were revealed on the retinal surface. Notably, RSCs migrated into the retinal ganglion cell layer and inner nuclear. Transplanted RSCs significantly elevated the amplitudes of a waves in retina injured eyes.

Conclusions: Embryonic RSCs have similar characteristics to neural stem cells. Transplantation of RSCs by intravitreal injection would be able to repair the damaged retina.

Keywords: Retinal stem cell, Retinal injury mice, Transplantation, Intravitreal injection

\section{Background}

Blindness eye diseases traditionally include cataract, corneal infection, and refractive stromal lesions. With the development of the basic research and clinical technologies, many of the oculopathy are becoming treatable. However, the spectrum for blindness eye disease is

\footnotetext{
*Correspondence: wanghong630223@163.com

${ }^{2}$ Department of Ophthalmology, Shandong Provincial Hospital Affiliated to Shandong First Medical University, No324, Jingwu Road, 250021 Jinan, China Full list of author information is available at the end of the article
}

changing remarkably. Currently, retinal disease is the leading cause of vision loss worldwidely [1]. Age-related macular degeneration (AMD) and retinitis pigmentosa (RP) are 2 representative retinal diseases [1]. Photoreceptors (PRs) is responsible for conversion of the light into electrical signals for further process and integration. Anatomically, PRs are in contact with the retinal pigment epithelium (RPE). RPE involves the transport of nutrients, the recycling of proteins, and the elimination of photoreceptor debris. RPE also secretes some growth

C C The Author(s). 2021 Open Access This article is licensed under a Creative Commons Attribution 4.0 International License, which permits use, sharing, adaptation, distribution and reproduction in any medium or format, as long as you give appropriate credit to the original author(s) and the source, provide a link to the Creative Commons licence, and indicate if changes were made. The images or other third party material in this article are included in the article's Creative Commons licence, unless indicated otherwise in a credit line to the material. If material is not included in the article's Creative Commons licence and your intended use is not permitted by statutory regulation or exceeds the permitted use, you will need to obtain permission directly from the copyright holder. To view a copy of this licence, visit http://creativecommons.org/licenses/by/4.0/ The Creative Commons Public Domain Dedication waiver (http://creativecommons.org/publicdomain/zero/1.0/) applies to the data made available in this article, unless otherwise stated in a credit line to the data. 
factors [2]. Therefore, dysfunctions or death of RPE cells can induce loss of PRs, leading to damage of vision and in some cases ultimately causing blindness. Of note, RPE are not able to endogenously regenerate [3]. Stem cells have the key ability to self-renew and differentiate to various types of cells. It has been shown that stem cells are becoming an attractive source of cell therapy in replacing or repairing damaged RPE and PRs [4]. Retinal stem cell therapy is one of the promising therapeutic alternatives to recover vision in patients with retinal disease $[2,5]$.

Three classes of stem or progenitor cells are utilized for cell therapy, including pluripotent stem cells (PSCs), fetal cells, and postnatal/adult cells. Cell-based therapies for retinal diseases that are currently under investigation usually use PSCs either embryonic stem cells (ESCs) or induced pluripotent stem cells (iPSCs) [3, 4, 6-9]. To date, a lot of studies have shown that stem cells or stem-cellderived cells improve the survival and function of host cells, not by producing the missing cells, but by secreting growth factors [10]. The transplanted cells can migrate and integrate into the various layers of the retina, potentially induced by neural differentiation stimulating factors [11-14]. iPSCs are similar to ESCs, but derived by dedifferentiating fully differentiated adult somatic cells (such as skin fibroblasts or white blood cells) into stem cells and then re-differentiating them into target cells [4]. Evidently, there are theoretical ethical advantages in using iPSCs for transplantation; but potential risks such as development of malignancy are present due to unknown mechanism of transformation and transcription factors $[15,16]$. Therapeutic RSCs can be delivered to the intravitreal space, posing a less risk than subretinal injection since the latter can result in retinal detachment [6]. As such, RSCs derived from ESCs appeared to have more benefits in the treatment of retinal disease. However, the application and the role of RSCs in the treatment of retinal degenerative diseases have not been fully revealed. At present, many issues in the application of RSCs need be explored and solved, such as the source of RSCs used for transplantation, transplantation methods, local survival periods and electrophysiological function maintenance, etc.

This study aimed to discuss the current stem cell transplantation approaches, and to investigate the feasibility and significance of embryonic RSCs transplantation by intravitreal delivery in a retinal damaged mice model. We found that embryo-derived RSCs have similar characteristics to neural stem cells (NSCs), which were evidenced by the presence of Pax6 and Nestin expressions. Transplanted RSCs were observed in all layers of the retina, suggesting that they have potential to function to repair the damaged retina. Accordingly, these findings herein provided theoretic evidence that facilitate the studies of RSCs transplantation in the treatment of retinal disease.

\section{Methods}

\section{Animals}

Healthy Kunming mice aged 5-6 weeks $(n=15 ; 3$ pregnant and 12 non-pregnant) were purchased from Beijing HuaFuKang Biotechnology Co., LTD (Beijing, China), and maintained on a regular diurnal lighting cycle (12:12 light: dark) with ad libitum access to food and water under specific-pathogen-free conditions at the Central Animal Care Services of Shandong University. Carbon dioxide inhalation was used for euthanasia. Mice were placed into a chamber initially containing room air with the lid closed, and then $100 \%$ compressed carbon dioxide was infused for $30 \mathrm{~s}$ at a displacement rate of $30 \%$ of the chamber volume per minute to induce a rapid anesthesia, followed by an increasing flow rate and continued exposure of $3 \mathrm{~min}$ for death induction. Upon completion of the procedure, death was confirmed by absence of all muscle activity and vital signs (no breath, mydriasis) for at least $30 \mathrm{~s}$ for each animal. This study was approved by the Shandong Provincial ENT Hospital (Shandong Provincial Western Hospital) Ethical Committee (Project No: XYK-20,200,701), and all procedures were conducted in accordance with the National Institutes of Health Guide for the Care and Use of Laboratory Animals.

\section{Preparation of retinal stem cells}

At the gestational age of 17 days, pregnant Kunming mice $(n=3)$ were euthanized, and embryos were immediately excised. As described previously [17-19], both the retina and ciliary body tissue including the pigmented layer-the ciliary marginal zone (CMZ) were cut into small pieces under a dissecting microscope for RSCs isolation (Fig. 1). The resulting tissues were digested using $100 \mathrm{U} / \mathrm{mL}$ collagenase (cat. no. C0130, Sigma-Aldrich, Shanghai, China) for $1 \mathrm{~h}$, followed by 0.25\% trypsin (cat. no. 15,050,057, Invitrogen, Shanghai, China) digestion for 15 min. Single cell was obtained by passing the suspension through use of a stainless-steel filter with $50 \mu \mathrm{m}$ porous. Subsequently, the cells were transferred to a $25 \mathrm{~mL}$ culture flask at an inoculum density of $5 \times 10^{4} / \mathrm{mL}$ in a $1: 1$ nutrient mixture of Dulbecco's modified Eagle's medium (DMEM) and F-12 (cat. no. 11,330,057, Gibco, Gaithersburg, MD, USA), supplemented with $1 \%$ B-27 (cat. no. 17,504,044, Gibco) and $20 \mathrm{ng} / \mathrm{ml}$ bFGF (cat. no. PHG0368, Gibco). Cells were then cultured at $37{ }^{\circ} \mathrm{C}$ under $5 \% \mathrm{CO}_{2}$. After 8 days of the culture, the primary suspensions of the cellular clusters were passaged in a 1:2 ratio following $1 \mathrm{~h}$ collagenase digestion and disruption as a result of being forced through a sterile syringe for 5 times. The process was repeated every 5-7 days. The sixth-generation cells were identified for RSCs using indirect immunofluorescence staining for Nestin and Pax6. 


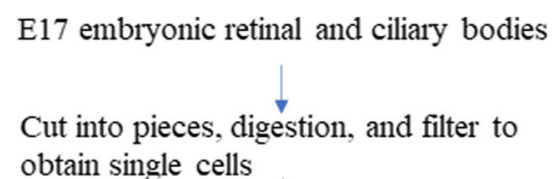

Culture for 8 days in DMEM/F-12 (1:1) plus B-27 and bFGF

- Increase of cell number by divisions

- Differentiation into RSCs

Passage in 1:2 every 5-7 days

The $6^{\text {th }}$-generation RSCs: label BrdU for transplantation

RSCs intravitreal injection to repair damaged retina

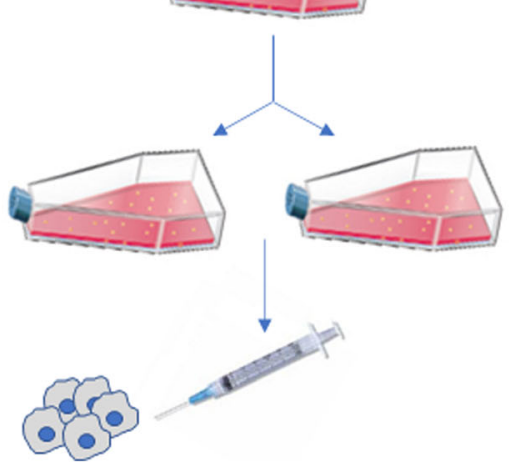

Under phase contrast microscope, check morphology of primary cultured cells at day 4 (Fig 2A)

Under phase contrast microscope, check morphology of the $4^{\text {th }}$ and $6^{\text {th }}$-generation cells cultured for 3 days (Fig 2B,C)

In the $6^{\text {th }}$-generation cells cultured for 3 days

- Check Pax6 and Nestin expressions (Fig 3)

- Check BrdU incorporation (Fig 5)

After 2 weeks, assess migration of injected $\mathrm{RSCs}$ in the damaged retina by checking BrdU, Pax6 and Nestin (Fig 6)

Fig. 1 Schematic of the preparation of retinal stem cell

\section{5-Bromo-2'-deoxyuridine (BrdU) labeling of RSCs}

RSCs from the sixth-generation cultures were added to Poly-L-lysine packaged sterilized culture dishes and cultured for 3 days. Thereafter, $200 \mu \mathrm{g} / \mathrm{ml}$ of BrdU solution (cat no. 19-160, Sigma-Aldrich) was added to the cells. The cells were cultured for 2 days in the presence of BrdU. Incorporation of BrdU was assessed using immunohistochemical staining.

\section{Optic nerve crush injury}

Twelve male Kunming mice aged 8 months were anesthetized with $2 \%$ pentobarbital sodium by intraperitoneal injection. This allowed us to drop tetracaine solution (cat. no. 4512, Sigma Pharmaceuticals, North Liberty, IA, USA) into the left eyes for surface anesthesia, and clamp optic nerve with a micro-artery clamp for $15 \mathrm{~s}$ at $2 \mathrm{~mm}$ behind the globe. The right eyes without any treatment were used as the controls. Retinal slicing and hematoxylin and eosin ( $\mathrm{H} \& \mathrm{E})$ histological staining was performed 1 week after procedure.

\section{Retinal stem cell transplantation}

The standard intravitreal injection for RSCs transplantation was used as described previously [20]. Twelve
Kunming male mice with left injured retina were randomly divided into RSCs injection group $(n=6)$ and PBS injection group $(n=6)$ in order to investigate the role of transplanted RSCs. Mice were anesthetized with $2 \%$ pentobarbital sodium by intraperitoneal injection, and the limbs and head were fixed well to allow access to the left (operative) eyes. Under a surgical microscope, a $10 \mu \mathrm{l}$ micro-syringe connected to a 30G needle was inserted into the vitreous cavity from the corneoscleral limbus of the left injured eye in 3 mice, and $2 \mu \mathrm{l}$ cell suspension of RSCs $\left(3 \times 10^{4}\right.$ cells $\left./ \mu \mathrm{l}\right)$ from the 6 th -generation cultures was carefully injected into the vitreous cavity. All injections were successful, which was verified by without bleeding after observing for $30 \mathrm{~s}$. The left injured retina in the other 6 mice were injected with $2 \mu$ of sterile PBS in the same manner, serving as the controls. After 2 weeks, mice were thereafter euthanized, and eyeballs were removed for immunohistochemical staining. Of note, the operator was unaware of the group allocation and disclosed it until results were obtained and analyzed.

\section{Electroretinogram (ERG)}

Electroretinogram (ERG) was performed to assess the electrical activity of the retina. The German RETLport visual physiological detection system was used for ERG detection. The recording electrode was a self-made 
acupuncture needle electrode, placed on the limbus, and the reference electrode and ground electrode were placed under the skin of the cheek and tail, respectively. Before the test, the mouse was placed in a dark room for half an hour to adapt to the dark. After the dark adaptation, the pentobarbital sodium was anesthetized intraperitoneally, the cheeks and tail hair were shaved, and the experiment was performed in an electrically shielded dark room. The passband was set at $5-30 \mathrm{~Hz}$, the impedance between the recording electrode and the reference electrode was $<5 \mathrm{~K} \Omega$; the sampling time was $250 \mathrm{~ms}$, and the waveform was superimposed and averaged for 200 times.

\section{H\&E staining}

H\&E staining was performed on the sections of paraffinembedded tissue according to the standard methods.

\section{Immunohistochemistry staining}

The sections of paraffin-embedded tissue were initially placed into a temperature-controlled chamber at $60{ }^{\circ} \mathrm{C}$ for $30 \mathrm{~min}$, followed by $10 \mathrm{~min}$-deparaffinization in xylene and a series of gradient ethanol solutions: $100 \%$ ethanol for $1 \mathrm{~min}, 95 \%$ for $1 \mathrm{~min}$, and $70 \%$ for $1 \mathrm{~min}$. After hydration in distilled water for $2 \mathrm{~min}$, the slices were immersed into $3 \%$ hydrogen peroxide for $15 \mathrm{~min}$ at room temperature to block endogenous peroxidase activity. For antigen retrieval, the slices were immersed into $2 \mathrm{~mol} / \mathrm{L}$ $\mathrm{HCl}$ for $1 \mathrm{~h}$ and then dried at room temperature, followed by $0.1 \mathrm{~mol} / \mathrm{L} \mathrm{NaOH}$ for $2 \mathrm{~s}$ and rinse with PBS for $2 \mathrm{~min}$. This step was repeated 3 times.

Subsequently, sections were stained with mouse antiBrdU antibody (1:100, cat. no. ab8152, Abcam, Cambridge, United Kingdom) overnight at $4{ }^{\circ} \mathrm{C}$. After rinsed 3 times with PBS, sections were incubated with sheep antimouse IgG solution (1:50, cat. no. ab6710, Abcam) at room temperature for $30 \mathrm{~min}$. 3,3 diaminobenzidine (DAB) coloration was performed under microscope, and hematoxylin was used to stain the nuclei for $3 \mathrm{~min}$. The specimens were then rinsed with water, dehydrated, and sealed for observation and imaging. Representative images were taken with optical microscope (Leica DM2500, Will and International Trade Co., LTD, Hong Kong).

\section{Indirect immunofluorescence staining}

Indirect immunofluorescence staining of Nestin and Pax6 were performed on cold acetone-fixed frozen tissue sections. After 3 washes with PBS, the sections were blocked in $10 \%$ goat serum for $1 \mathrm{~h}$ to decrease background signal. Rabbit anti-Pax6 (1:100, cat. no. ab195045, Abcam) and mouse anti-Nestin (1:100, cat. no. ab6320, Abcam) were added and incubated overnight at $4{ }^{\circ} \mathrm{C}$ in a wet box. After 5 washes with PBS, Alex488 and 594 conjugated goat anti-rabbit or mouse IgG (1:2, 000, cat. no. A-11,034, cat. no. A-11,032, Invitrogen) were applied at $37^{\circ} \mathrm{C}$ for $1 \mathrm{~h}$ in the dark room. Notably, the staining when the primary antibodies were omitted and only the secondary antibodies were applied were used as the blank controls. After 5 washes with PBS, DAPI was added and incubated in dark for $2 \mathrm{~min}$. The specimens were then sealed with glycerin and immediately observed and photographed under a fluorescence microscope (BD-YG500, Shenzhen Boshida Optical Instrument Co., Ltd., Shenzhen, China).

\section{Statistical analysis}

Data are shown as mean \pm standard deviation. Two tailed $t$ test was performed through use of Graphpad Prism 8.2.1 software (GraphPad Software, Inc., San Diego, CA, USA). $P<0.05$ is considered as having significant differences.

\section{Results}

Culture and identification of retinal stem cells

Mouse retinal and ciliary bodies from E17 embryos were used to isolate RSCs. Under phase contrast microscope, most of the primary cultured cells showed fusiform or round phenotype, and a few of cells were long and thin strip cells at day 4 after culture (Fig. 2a). The cell vitality was $40-50 \%$. For the 4 th -generation cells, 3 days after culture, the cell density was increased, and most of the cells were round (Fig. 2b). After extending to the sixth generation, the cells grew well, and round cells increased obviously (Fig. 2c). This indicated that the cultured cells possessed the morphological characteristics of RSCs.

The sixth-generation cells were cultured, and after 3 days, Pax6 and Nestin were stained using indirect immunofluorescence staining for verification of RSCs. It has been demonstrated that Pax6 is required for the multipotent state of retinal progenitor cells [21]. Nestin, a cytoskeletal intermediate filament, is initially characterized in neural stem cells. However, current extensive evidence suggested that Nestin plays an essential role in stem cell functions, including self-renewal, differentiation and migration [22]. Therefore, Pax6 and Nestin are commonly used as the markers of retinal stem cells. Our results showed that Nestin was predominantly localized in cytoplasm, and Pax6 was mainly observed in nuclei (Fig. 3), suggesting that the 6th -generation cells were RSCs.

\section{Alterations of retinal histology and ERG in the mice with optic nerve crush injury}

Optic nerve crush injury model was induced by clamping optic nerve through use of a micro-artery clamp. Clamping decreased and/or blocked the blood supply of the central retinal artery running in the optic nerve, leading to retinal ischemia, cellular apoptosis and tissue necrosis. One week after the operation, retinal structure was checked 

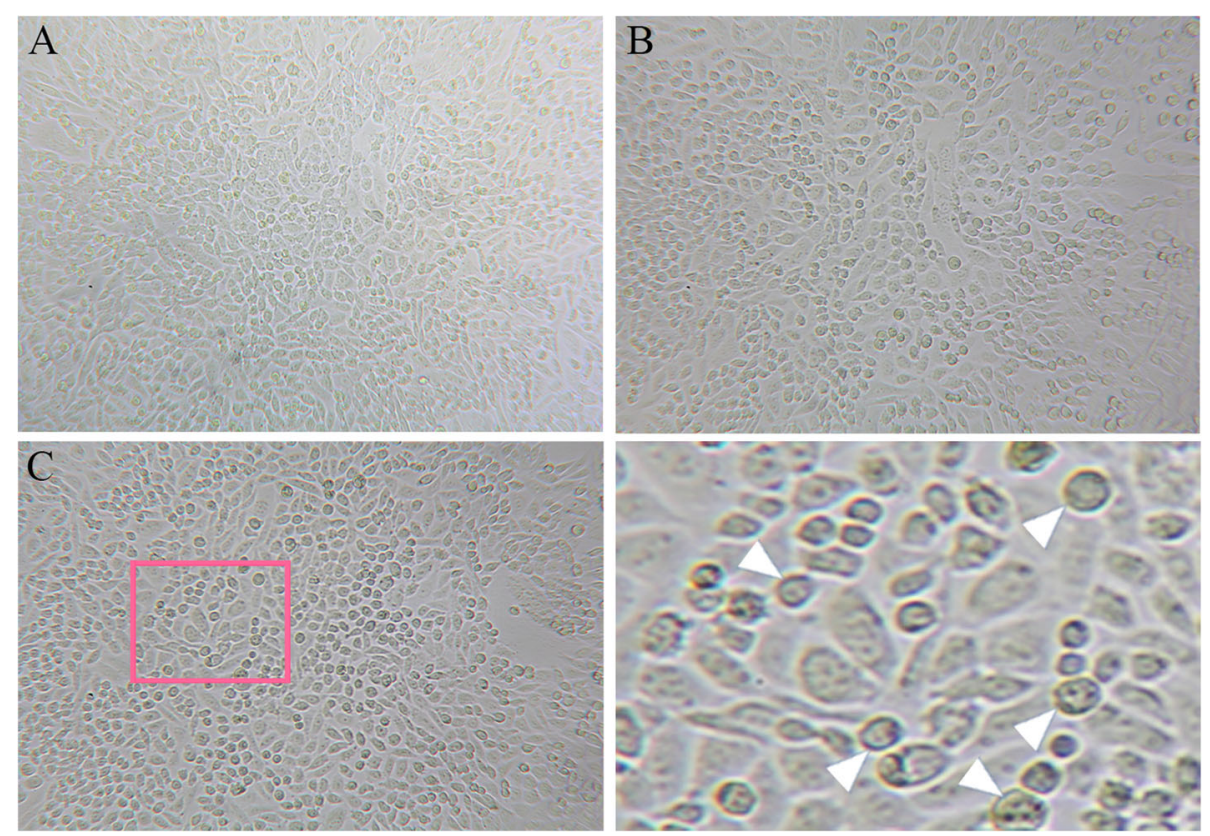

Fig. 2 Generation of mouse retinal stem cells. Retinal stem cells were isolated from embryonic E17 retinal and ciliary bodies. a Phase contrast imaging of primary cultured cells at 4 days. Most of the cells showed fusiform or round phenotype. $\mathbf{b}$ Three days after extending to the 4 th generation, cell densities increased, and the majority of cells were round. $\mathbf{c}$ After extending to the 6th generation, the cells grew well, and round cell density increased as indicated by the arrowhead. Magnification: $\times 200$ for all images. A high-power view of the selected area was presented on the right

using H\&E staining. Images captured at high magnifications with a microscope showed that the retinal cell nuclei of the control group were well arranged with normal morphologies. Nevertheless, cellular nuclear disintegration and fragmentation were observed in the retinal tissue of the injured group. Of note, the morphological changes of the retinal granular layer in the injured group progressed over the periods of clamping time (in seconds). At $15 \mathrm{~s}$ following clamping, obvious pathological changes were found and characterized by the disordered granular layer cell structure, the ruptured cell membranes, and the collapsed cytoplasm and nuclei, especially in the outer granular layer. At $60 \mathrm{~s}$, the cellular morphologies were dramatically damaged (Fig. 4). As the degree of cell damage at $15 \mathrm{~s}$ was closer to that seen in the patients with retinal diseases, we chose $15 \mathrm{~s}$ for optic nerve clamping time to establish the retinal damage model.

In addition, the data from ERG showed that the amplitudes of a wave, particularly the $\mathrm{b}$ wave were significantly lower in the injury group than that in the control group (Table 1). This indicates that both the outer and the inner nuclear layers was damaged by the clamping procedure.

\section{Retinal stem cells transplantation for repair of the damaged retina}

The 6th -passage RSCs labeled with BrdU were used for transplantation. Since it can be incorporated into the newly synthesized DNA of replicating cells, BrdU is frequently used in analysis of neural stem cell biology, in particular to label and to fate-map dividing cells [23]. Firstly, incorporation of BrdU was assessed on the fixed cells. Immunohistochemical staining show that approximately $90 \%$ of RSCs were labeled with BrdU, evidenced by brownish staining in nuclei (Fig. 5).

RSC suspensions were then transplanted into the damaged retina using the intravitreal injection method. Two weeks post-operation, pathological sections were stained for BrdU, Pax6 and Nestin. In the transplanted retina with RSCs, nuclear staining of BrdU and Pax6, and cytoplasmic staining of Nestin, were revealed on the surface of retina, predominantly between the retinal ganglion layer and inner nuclear layer (Fig. 6a, b). Notably, a few of positive stains were also detected in the outer nuclear layer in the damaged eyes that accepted transplantation of RSCs (Fig. 6a, b). In the control eyes, all layers of the retina were not positively stained. Representative images from one of the retinal injured mice with RSCs or PBS injections were provided.

To assess if the transplanted RSCs have the ability to repair the electrical activity of the damaged retina, we performed ERG experiments in mice with optic nerve crush injury receiving either RSCs or PBS transplant. Compared to the PBS transplantations, RSCs 


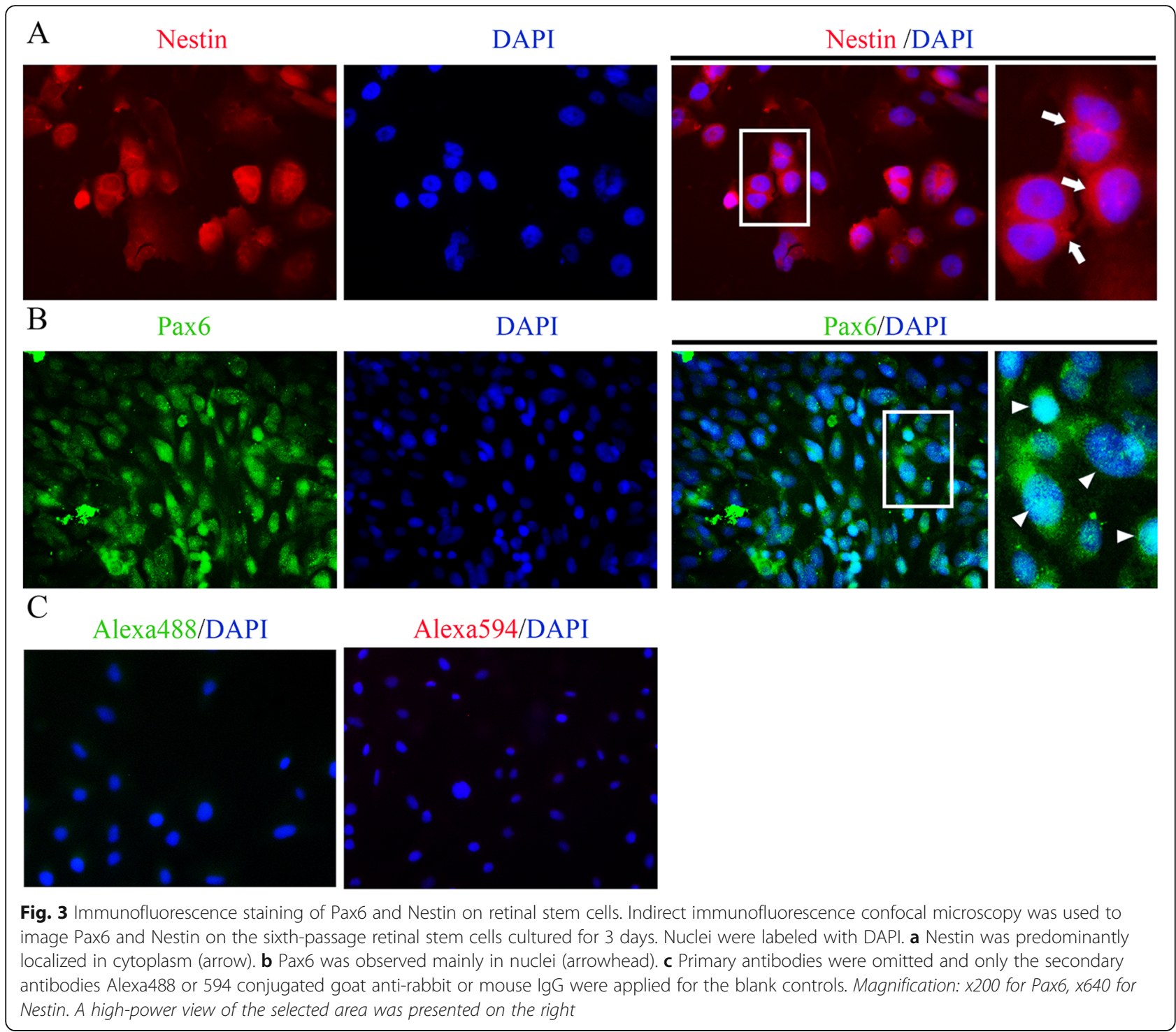

significantly augmented the amplitudes of a wave (Fig. 6c). The b waves did not show remarkable differences between RSCs and PBS transplanted retina. This indicated that after RSCs transplantation, the function of the outer layer of the injured retina recovered at least to a certain extent. Nevertheless, transplanted RSCs did not improve the function of the inner layer of the retina, possibly associated with the differentiation of RSCs and the limited time we had to observe.

\section{Discussion}

Stem cell engineering has opened a new avenue for repairing damaged nervous tissues. Following transplantation of stem cells into eyes, they further integrate into the retinal microenvironment, and then proliferate and differentiate into target cells, to regenerate the damaged neurons [6]. This offers recovery and reconstruction of the retinal function, with opportunities to treat irreversible blindness ophthalmopathy.

Previous work has demonstrated that the injection of ESCs into the subretinal space of rats effectively alleviated photoreceptor cell degeneration and death [24]. NSCs have been found in the embryonic nervous system and in certain parts of the adult brain. Due to continuous self-renewal and proliferative ability, these cells can differentiate into specific neurons and glial cells. Recently, it has been reported that NSCs were successfully integrated into the various layers of the retina [25-27]. The major challenge is how the NSCs differentiate into mature retinal cells. Some studies have shown that differentiation was associated with the growth environment of the cell [28-30]. In addition, there is an ongoing clinical trial from ReNeuron about the application of the human retinal progenitor cell therapy in the treatment of 


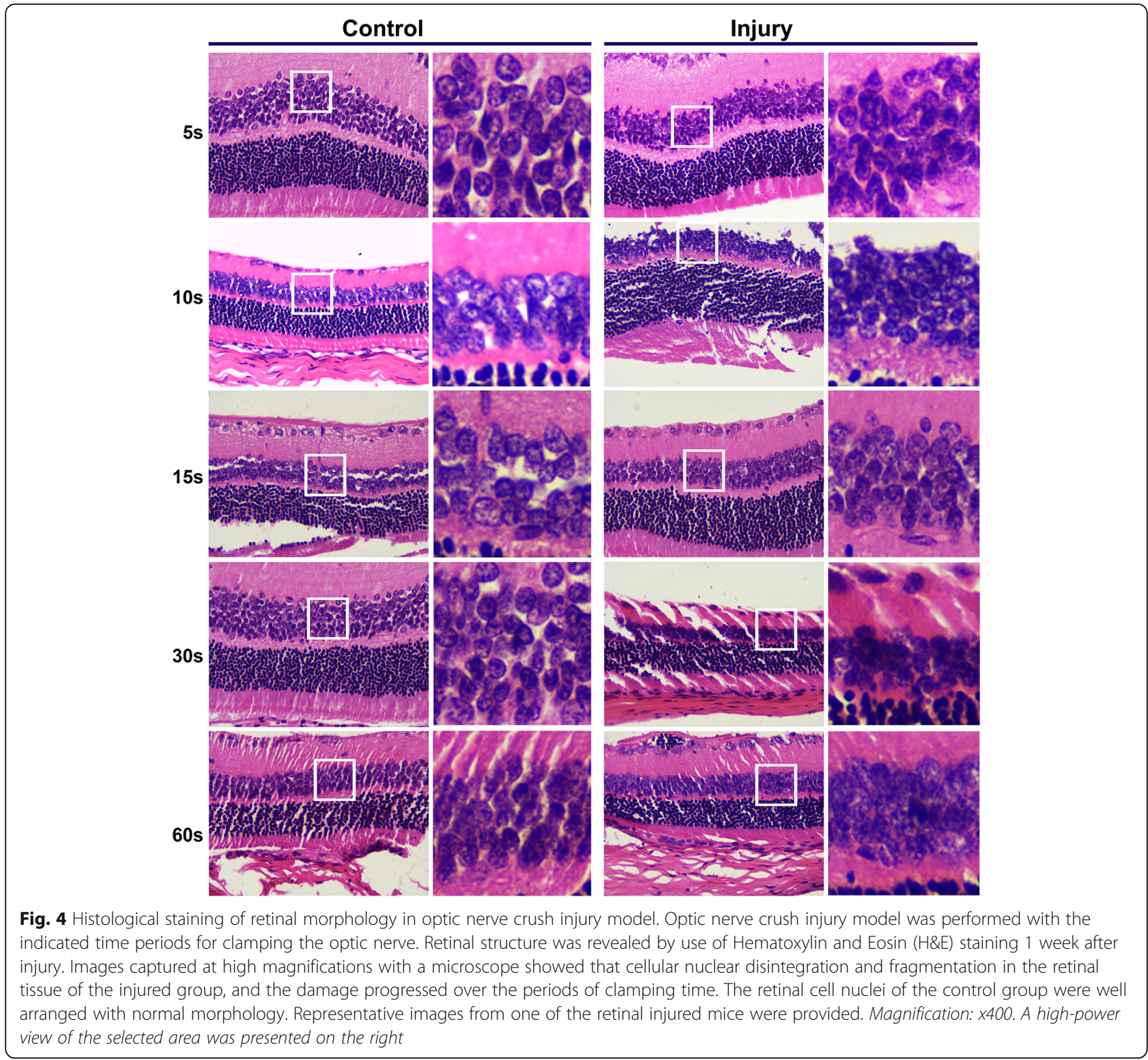

patients with retinitis pigmentosa (NCT02464436). In the present study, we isolated, cultured, and propagated mouse RSCs from E17 embryos. After extending to the 4th generation, the cells presented with the phenotype of RSCs. To further verify if these cells were stem cells and had the characteristics of NSCs, we stained the stem cell marker Pax6 and NSC-specific marker Nestin [21,

Table 1 Comparison of the amplitudes of the ERG between the injury and the control groups

\begin{tabular}{lllr}
\hline & Injury $(\boldsymbol{n}=\mathbf{6})$ & CTL $(\boldsymbol{n}=\mathbf{6})$ & P value \\
\hline a wave $(\mu \mathrm{V})$ & $-38.74 \pm 5.28$ & $-64.42 \pm 5.29$ & 0.000 \\
b wave $(\mu \mathrm{V})$ & $87.72 \pm 10.86$ & $168.4 \pm 13.92$ & 0.000 \\
\hline
\end{tabular}

22]. Cultured RSCs not only had stem cell morphologies under phase contrast microscope, but also highly expressed Pax6 and Nestin, indicating that the cultured RSCs belonged to the NSC family. Therefore, embryonic RSCs might be becoming prospective cells for retinal transplantation.

Considering the RSC as the most suitable seed cell, we further examined the feasibility of RSCs transplantation in the treatment of the damaged retina. In general, there are currently 2 types of transplantation methods: subretinal space injection and vitreous cavity injection $[6,31]$. Maintaining intraocular structure and preventing immune rejection is crucial for the survival of transplanted cells. In the case of subretinal space injection, it is often difficult to avoid blood retinal barrier disruption within 


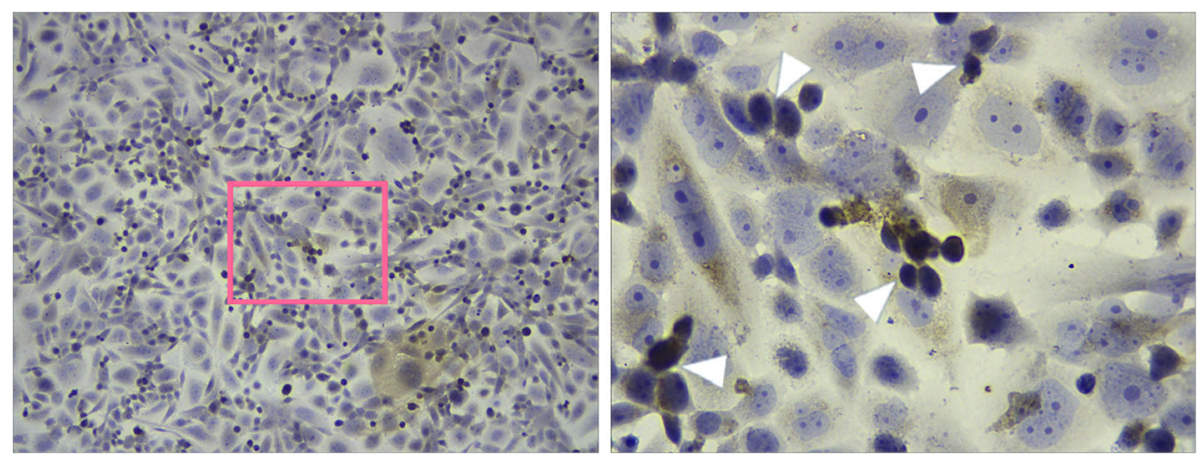

Fig. 5 Immunohistochemical staining of BrdU on retinal stem cells. The sixth-generation retinal stem cells were cultured for 3 days after passaging. Immunohistochemical staining of BrdU were then performed on fixed cells. BrdU was incorporated into most of retinal stem cells, evidenced by yellow-brown nuclei (arrowhead). Magnification: $\times 200$. A high-power view of the selected area was presented on the right

the eyeball, which can cause severe swelling as well as degeneration and/or death of transplanted cells [31]. The intravitreal injection is a simplest feasible way in clinical practice for intraocular medication, which allows the eyeball to remain intact and reduces the occurrence of damage to the retina barrier. Moreover, this method offers a clear field of view during operation [6,31,32]. Although both of these grafting methods are capable of integrating seed cells into the retina, some studies recently compared the 2 methods, showing that trauma induced by the subretinal injection is considerably greater leading to retinal detachment [17]. Therefore, the intravitreal injection method is commonly preferred for delivery of medication or stem cell. In our study, we delivered BrdU-labeled RSCs into vitreous cavity for cell transplantation. Upon examination of the retina postoperation till 2 weeks no infections or bleeding were observed. Close observation of sectioned specimens revealed a complete intraocular structure with apparent anatomic arrangement. These findings suggest that intravitreal injection is a safe delivery way with less trauma for transplantation.

In order to gain an insight into the cell arrangement, we investigated the effect of transplanted RSCs on the retinal neuron composition. Retinal neurons are typically divided into 3 layers: ganglion cells, bipolar cells, and photoreceptor cells, from the inner to outermost layer, respectively [33]. Photoreceptor cells convert light stimuli into nerve impulses, the bipolar cells transfer nerve impulses to ganglion cells, and the nerve impulses transfer through the ganglion cells nerve fiber to the optical center, which produces the visual [33]. As the retinal ganglion cells are associated to nerve fibers, any mechanical damage to the optic nerve can block axoplasmic transport of the ganglion cells, leading to direct impairment of ganglion cell nutrition [33]. The pathology of such retinal damage and visual function lesions are mimicked by ring clamping of the optic nerve [34].
Here, we firstly performed optic nerve crush injury model by ring clamping the optic nerve. One week after operation, images captured at high magnifications with a microscope showed nuclear disintegration and fragmentation in the retinal tissue of the injured group, and the damage progressed over the periods of clamping time. Retinal ganglion cells and other inner retinal cells are mainly affected by ischemic injury since the central retinal artery mainly supply inner two thirds of retina. Consistently, the findings from ERG showed that the amplitudes of a wave, in particular the b wave, was significantly decreased in the injured retina compared to the control. Therefore, more dramatic injury of the inner nuclear layers was disclosed following optic nerve crush. We then attempted to transplant the BrdU-labeled RSCs in close proximity to the ganglion cell layer, which allowed us to assess the effect of the damaged optic nerves directly. Notably, the percentage of the BrdUpositive RSCs was greater than $90 \%$, indicating that almost all the RSCs were labeled. Characterization of transplanted tissues was thereafter carried out after 2 weeks. Our results showed that the BrdU-positive cells were present in vitreous cavity, the retinal surface and the ganglion cell layer, indicating a successful transplantation of the RSCs and their entry into the damaged retina. This is further demonstrated by the presence of Pax6 and Nestin-positive cells in the retina, predominantly between the retinal ganglion layer and inner nuclear layer. Morphological evaluation also suggested that the transplanted RSCs were integrated into the host retina, implying its potential to substitute for the damaged cells. This observation was consistent with the previous reports [35, 36]. The preliminary data from ERG further demonstrated the potential repair ability of RSCs, which was restricted in the outer layer of the damaged retina. It may be associated with the differentiation of RSCs and the limited time we had to observe. Additionally, a large number of RSCs were injected into the vitreous cavity, 


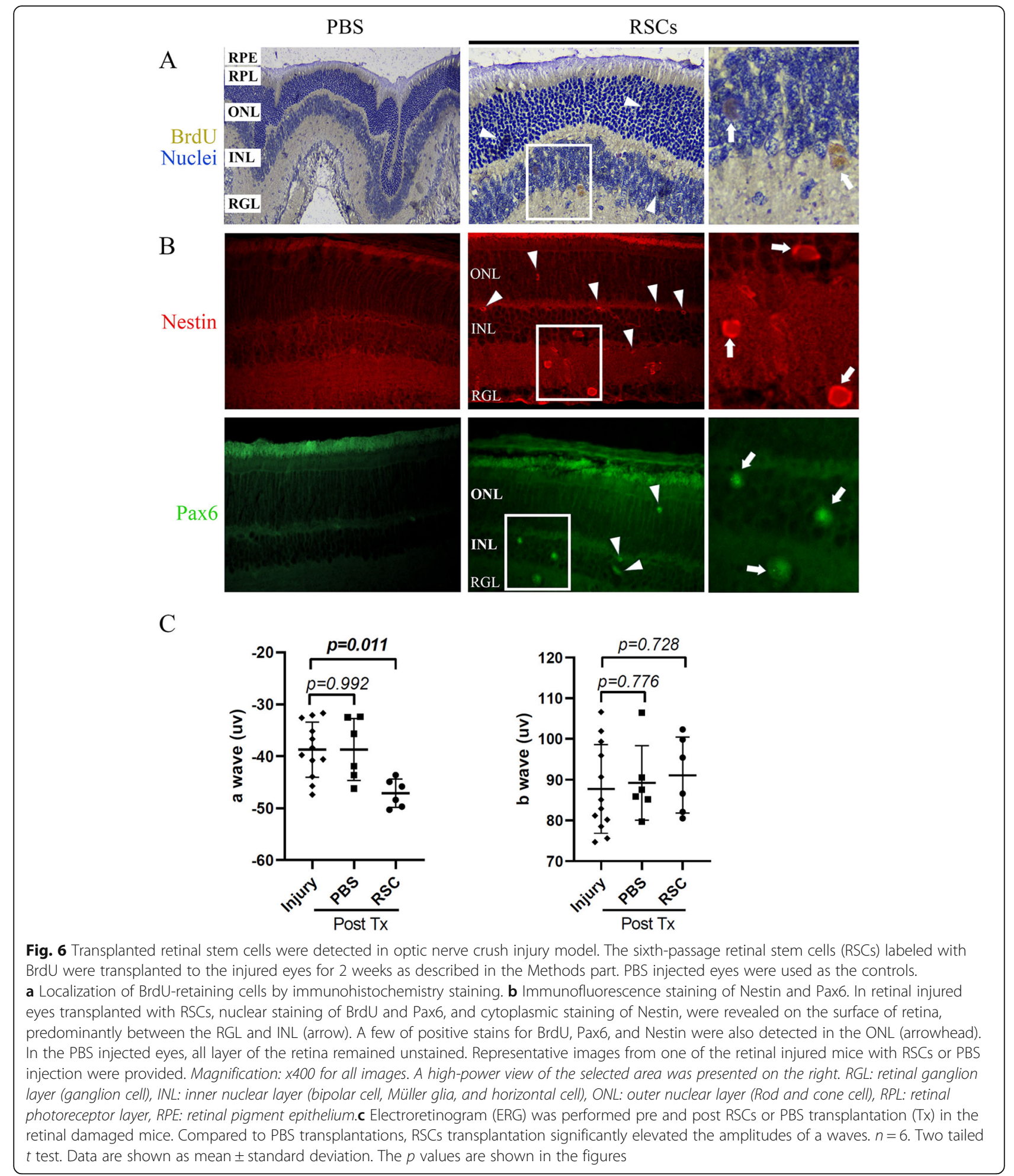

and only a few cells were observed to survive in the vitreous and retina. It was speculated that it might be related to cellular apoptosis induced by autoimmune response. How to keep and increase RSCs survival in living tissues is 1 of the major challenges in the research of
RSCs transplantation. Whether RSCs can smoothly migrate or differentiate into inner retinal cells remains to be further studied. Notably, the observation time of the current study was relatively short, and the key synaptic link may not have been developed yet. 
Several studies have shown that the transplanted NSCs in retinas can partially differentiate into neurons [3739], but generally depend on microenvironment of the host $[40,41]$. In addition, it has been reported that this differentiation was also related to the host age. Li N et al. showed that the integration ability of stem cells transplanted into the vitreous cavity of rats decreased with the increase of host age [42]. Nevertheless, this phenomenon may be associated with a range of other factors such as the existence of a large number of undifferentiated cells in the neonatal host, an imperfect barrier function, and some growth factors promoting cell migration [43]. Furthermore, it has been shown that when the graft contains more mature cells it easily forms aggregates of cells or rosettes, which disrupt the integration of graft and host and thus prevent the transplanted cells from reconstructing the retinal function [44]. However, embryonic cells or stem cells are not easy to form a rosette even with a high number [45]. As such, it is evident that the purity of transplanted cells is extremely important to allow differentiation into functional target cells even if ESCs or embryonic RSCs are used.

\section{Conclusions}

Our findings suggested that embryo-derived RSCs exhibited similar properties to those of NSCs, and that transplantation of RSCs had potential to repair the damaged retina. Additionally, intravitreal transplantation of RSCs was a simple feasible delivery way in the treatment of retinal disease. However, many issues about RSCs transplantation still require further extensive investigation, such as the key factors for transplanted RSCs survival, the role of the transplanted RSCs-produced cytokines in the repairment of damaged retina, and the factors that can promote the differentiation of the transplanted RSCs into mature retinal functional cells.

\section{Abbreviations \\ AMD: Age-related macular degeneration; RP: Retinitis pigmentosa; PR: Photoreceptors; RPE: Retinal pigment epithelium; PSCs: Pluripotent stem cells; ESCs: Embryonic stem cells; iPSCs: Induced pluripotent stem cells; RSCs: Retinal stem cells; CMZ: Ciliary marginal zone; DMEM: Dulbecco's modified Eagle's medium; BrdU: 5-bromo-2'-deoxyuridine; PLL: Poly-L-lysine; H\&E: Hematoxylin and eosin; RNL: Retinal neurosensory layer; RPE: Retinal pigment epithelium; CL: Choroid layer; NSCs: Neural stem cells}

\section{Acknowledgements}

We thank Drs. Hu Huang and Jing Ji for technical support in cell cultures.

\section{Authors' contributions}

Involved in the design of the study (HW); conduct of the study (XF, PC, XZ and JW); collection, management, and analysis of the data (XF and PC); preparation of the manuscript (XF); and critical revision of the manuscript (XF and $\mathrm{HW}$ ). All authors have read and approved the final manuscript.

\section{Funding}

This work was supported by the Shandong Province Technology Government Agency (No. 013GSF31804) and Shandong Province Hospital and Research Institute of EENT. The sponsors and funding organizations played no role in the design of the study and collection, analysis, and interpretation of data and in writing the manuscript.

\section{Availability of data and materials}

All the data used to support the findings of this study are included within the article and are available from corresponding author by a reasonable request.

\section{Ethics approval and consent to participate}

This study was approved by Shandong Provincial ENT Hospital (Shandong Provincial Western Hospital) Ethical Committee (Project No: XYK-20200701), and all procedures were conducted in accordance with the National Institutes of Health Guide for the Care and Use of Laboratory Animals.

\section{Consent for publication}

Not applicable.

\section{Competing interests}

The authors declare that they have no competing interests.

\section{Author details}

${ }^{1}$ Department of Ophthalmology, Shandong Provincial Western Hospital, Shandong Provincial ENT Hospital, Jinan, China. ${ }^{2}$ Department of Ophthalmology, Shandong Provincial Hospital Affiliated to Shandong First Medical University, No324, Jingwu Road, 250021 Jinan, China.

Received: 18 July 2020 Accepted: 26 December 2020

Published online: 09 January 2021

\section{References}

1. Sloan FA, Hanrahan BW. The Effects of Technological Advances on Outcomes for Elderly Persons With Exudative Age-Related Macular Degeneration. JAMA Ophthalmol. 2014;132:456-63.

2. M'Barek KB, Monville C. Cell Therapy for Retinal Dystrophies: From Cell Suspension Formulation to Complex Retinal Tissue Bioengineering. Stem Cells Int. 2019;2019:4568979.

3. Lamba D, Karl M, Reh T. Neural Regeneration and Cell Replacement: A View From the Eye. Cell Stem Cell. 2008;2:538-49.

4. Zakrzewski W, Dobrzyński M, Szymonowicz M, Rybak Z. Stem Cells: Past, Present, and Future. Stem Cell Res Ther. 2019;10:68.

5. M'Barek KB, Habeler W, Monville C. Stem Cell-Based RPE Therapy for Retinal Diseases: Engineering 3D Tissues Amenable for Regenerative Medicine. Adv Exp Med Biol. 2018;1074:625-32.

6. Singh MS, Park SS, Albini TA, Canto-Soler MV, Klassen H, MacLaren RE, et al. Retinal stem cell transplantation: Balancing safety and potential. Prog Retin Eye Res. 2020;75:100779.

7. Drukker M, Katchman H, Katz G, Even-Tov Friedman S, Shezen E, Hornstein E, et al. Human embryonic stem cells and their differentiated derivatives are less susceptible to immune rejection than adult cells. Stem Cells. 2006;24: 221-9.

8. Takahashi K, Tanabe K, Ohnuki M, Narita M, Ichisaka T, Tomoda K, et al. Induction of pluripotent stem cells from adult human fibroblasts by defined factors. Cell. 2007:131:861-72.

9. Yu J, Vodyanik MA, Smuga-Otto K, Antosiewicz-Bourget J, Frane JL, Tian S, et al. Induced pluripotent stem cell lines derived from human somatic cells. Science. 2007;318:1917-20.

10. Otani A, Dorrell Ml, Kinder K, Moreno SK, Nusinowitz S, Banin E, et al. Rescue of retinal degeneration by intravitreally injected adult bone marrow-derived lineage-negative hematopoietic stem cells. J Clin Invest. 2004;114:765-74.

11. Mead B, Hill LJ, Blanch RJ, Ward K, Logan A, Berry M, Leadbeater W, et al. Mesenchymal stromal cell-mediated neuroprotection and functional preservation of retinal ganglion cells in a rodent model of glaucoma. Cytotherapy. 2016;18:487-96.

12. Jeon $\mathrm{S}, \mathrm{Oh} \| \mathrm{H}$. Regeneration of the retina: toward stem cell therapy for degenerative retinal diseases. BMP Rep. 2015;48:193-9.

13. Schwartz SD, Tan G, Hosseini H, Nagiel A. Subretinal transplantation of embryonic stem cell-derived retinal pigment epithelium for the treatment of macular degeneration: an assessment at 4 years. Invest Ophthalmol Vis Sci. 2016:57:15-8. 
14. Seiler MJ, Lin RE, McLelland BT, Mathur A, Lin B, Sigman J, et al. Vision recovery and connectivity by fetal retinal sheet transplantation in an immunodeficient retinal degenerate rat model. Invest Ophthalmol Vis Sci. 2017:58:614-30.

15. Cramer AO, MacLaren RE. Translating induced pluripotent stem cells from bench to bedside: application to retinal diseases. Curr Gene Ther. 2013;13: 139-51

16. MacLaren RE, Bennett J, Schwartz SD. Gene Therapy and Stem Cell Transplantation in Retinal Disease: The New Frontier. Ophthalmology. 2016; 123:98-106.

17. Eiraku M, Sasai Y. Mouse embryonic stem cell culture for generation of three-dimensional retinal and cortical tissues. Nat Protoc. 2011;7:69-79.

18. Song MJ, Bharti K. Looking into the future: Using induced pluripotent stem cells to build two and three dimensional ocular tissue for cell therapy and disease modeling. Brain Res. 2016;1638:2-14.

19. Sun J, Mandai M, Kamao H, Hashiguchi T, Shikamura M, Kawamata S, et al. Protective effects of human iPS-derived retinal pigmented epithelial cells in comparison with human mesenchymal stromal cells and human neural stem cells on the degenerating retina in rd1 mice. Stem Cells. 2015;33: 1543-53.

20. Mead B, Logan A, Berry M, Leadbeater W, Scheven BA. Intravitreally transplanted dental pulp stem cells promote neuroprotection and axon regeneration of retinal ganglion cells after optic nerve injury. Invest Ophthalmol Vis Sci. 2013;54:7544-56.

21. Marquardt T, Ashery-Padan R, Andrejewski N, Scardigli R, Guillemot F, Gruss P. Pax6 is required for the multipotent state of retinal progenitor cells. Cell. 2001;105:43-55.

22. Bernal A, Arranz L. Nestin-expressing progenitor cells: function, identity and therapeutic implications. Cell Mol Life Sci. 2018;75:2177-95.

23. Lehner B, Sandner B, Marschallinger J, Lehner C, Furtner T, Couillard-Despres $\mathrm{S}$, et al. The dark side of BrdU in neural stem cell biology: detrimental effects on cell cycle, differentiation and survival. Cell Tissue Res. 2011;345: 313-28

24. Amirpour N, Karamali F, Rabiee F, Rezaei L, Esfandiari E, Razavi S, et al. Differentiation of human embryonic stem cell-derived retinal progenitors into retinal cells by Sonic hedgehog and/or retinal pigmented epithelium and transplantation into the subretinal space of sodium iodate-injected rabbits. Stem Cells Dev. 2012;21:42-53.

25. Kruczek K, Gonzalez-Cordero A, Goh D, aeem A, Jonikas M, Blackford SJ, et al. Differentiation and transplantation of embryonic stem cell-derived cone photoreceptors into a mouse model of end-stage retinal degeneration. Stem Cell Reports. 2017:8:1659-74.

26. Chao JR, Lamba DA, Klesert TR, Torre A, Hoshino A, Taylor RJ, et al. Transplantation of human embryonic stem cell-derived retinal cells into the subretinal space of a non-human primate. Transl Vis Sci Technol. 2017;6:4.

27. Petrus-Reurer S, Bartuma H, Aronsson M, Westman S, Lanner F, André H, et al. Integration of subretinal suspension transplants of human embryonic stem cell-derived retinal pigment epithelial cells in a large-eyed model of geographic atrophy. Invest Ophthalmol Vis Sci. 2017;58:1314-22.

28. Sigulinsky CL, German ML, Leung AM, Clark AM, Yun S, Levine EM. Genetic chimeras reveal the autonomy requirements for $\mathrm{Vs} \times 2$ in embryonic retinal progenitor cells. Neural Dev. 2015;10:2-22.

29. Fernandez-Alonso R, Martin-Lopez M, Gonzalez-Cano L, Garcia S, Castrillo F, Diez-Prieto I, et al. P73 is required for endothelial cell differentiation, migration and the formation of vascular networks regulating VEGF and TGF $\beta$ signaling. Cell Death Differ. 2015;22:1287-99.

30. Besser $M$, Jagatheaswaran $M$, Reinhard J, Schaffelke P, Faissner A. Tenascin C regulates proliferation and differentiation processes during embryonic retinogenesis and modulates the de-differentiation capacity of Muller glia by influencing growth factor responsiveness and the extracellular matrix compartment. Dev Biol. 2012;369:163-76.

31. Wilson DJ, Neuringer M, Stoddard J, Renner LM, Bailey S, Lauer A, et al. SUBRETINAL CELL-BASED THERAPY: An Analysis of Surgical Variables to Increase Cell Survival. Retina. 2017;37:2162-66.

32. Satarian L, Nourinia R, Safi S, Kanavi MR, Jarughi N, Daftarian N, et al. Intravitreal Injection of Bone Marrow Mesenchymal Stem Cells in Patients with Advanced Retinitis Pigmentosa; a Safety Study. J Ophthalmic Vis Res. 2017;12:58-64.

33. Lamb TD, Collin SP, Pugh EN Jr. Evolution of the vertebrate eye: opsins, photoreceptors, retina and eye cup. Nat Rev Neurosci. 2007:8:960-76.
34. Li Y, Schlamp CL, Nickells RW. Experimental induction of retinal ganglion cell death in adult mice. Invest Ophthalmol Vis Sci. 1999;40:1004-8.

35. Zhu D, Xie M, Gademann F, Cao J, Wang P, Guo Y, et al. Protective effects of human iPS-derived retinal pigmented epithelial cells on retinal degenerative disease. Stem Cell Res Ther. 2020;11:98.

36. Shrestha R, Wen YT, Tsai RK. Effective Differentiation and Biological Characterization of Retinal Pigment Epithelium Derived from Human Induced Pluripotent Stem Cells. Curr Eye Res. 2020:1-13. doi: https://doi.org/ 10.1080/02713683.2020.1722180

37. Iwai T, Saitoh A, Yamada M, Takahashi K, Hashimoto E, Ukai W, et al. Rhotekin modulates differentiation of cultured neural stem cells to neurons. J Neurosci Res. 2012;90:1359-66.

38. Brilli E, Reitano E, Conforti P, Conforti P, Gulino R, Consalez GG, et al. Neural stem cells engrafted in the adult brain fuse with endogenous neurons. Stem Cells Dev. 2013;22:538-47.

39. Efthymiou A, Shaltouki A, Steiner JP, Jha B, Heman-Ackah SM, Swistowski A, et al. Functional screening assays with neurons generated from pluripotent stem cell-derived neural stem cells. J Biomol Screen. 2014;19:32-43.

40. West EL, Pearson RA, Duran Y, Gonzalez-Cordero A, MacLaren RE, Smith AJ, et al. Manipulation of the recipient retinal environment by ectopic expression of neurotrophic growth factors can improve transplanted photoreceptor integration and survival. Cell Transplant. 2012;21:871-87.

41. Chen F, Wang H, Xiang X, Yuan J, Chu W, Xue X, et al. Curcumin increased the differentiation rate of neurons in neural stem cells via wnt sigaling in vitro study. J Surg Res. 2014;192:298-304.

42. Li N, Li XR, Yuan JQ. Effects of bone-marrow mesenchymal stem cells transplanted into vitreous cavity of rat injured by ischemia/reperfusion. Graefes Arch Clin Exp Ophthalmol. 2009;247:503-14.

43. Osakada F, Hirami Y, Takahashi M. Stem cell biology and cell transplantation therapy in the retina. Biotechnol Genet Eng Rev. 2010;26:297-334.

44. Klassen H, Kiilgaard JF, Warfvinge K, Samuel MS, Prather RS, Wong F, et al. Photoreceptor Differentiation following Transplantation of Allogeneic Retinal Progenitor Cells to the Dystrophic Rhodopsin Pro347Leu Transgenic Pig. Stem Cells Int. 2012;2012:939801.

45. Erceg S, Ronaghi M, Stojković M. Human embryonic stem cell differentiation toward regional specific neural precursors. Stem Cells. 2009;27:78-87.

\section{Publisher's Note}

Springer Nature remains neutral with regard to jurisdictional claims in published maps and institutional affiliations.

\section{Ready to submit your research? Choose BMC and benefit from:}

- fast, convenient online submission

- thorough peer review by experienced researchers in your field

- rapid publication on acceptance

- support for research data, including large and complex data types

- gold Open Access which fosters wider collaboration and increased citations

- maximum visibility for your research: over $100 \mathrm{M}$ website views per year

At $\mathrm{BMC}$, research is always in progress.

Learn more biomedcentral.com/submissions 